Article

\title{
Synthesis and Biological Evaluation of Novel Benzothiazole Derivatives as Potential Anticonvulsant Agents
}

\author{
Da-Chuan Liu, Hong-Jian Zhang, Chun-Mei Jin * and Zhe-Shan Quan *
}

Key Laboratory of Natural Resources and Functional Molecules of the Changbai Mountain, Affiliated Ministry of Education, College of Pharmacy, Yanbian University, Yanji 133002, China; 2013001048@ybu.edu.cn (D.-C.L.); zhjzhishixiang@163.com (H.-J.Z.)

* Correspondence: cmjin@ybu.edu.cn (C.-M.J.); zsquan@ybu.edu.cn (Z.-S.Q.);

Tel./Fax: +86-433-243-6017 (C.-M.J.); +86-433-243-6020 (Z.-S.Q.)

Academic Editors: Shufeng Zhou and Wei-Zhu Zhong

Received: 6 January 2016 ; Accepted: 25 January 2016 ; Published: 29 February 2016

\begin{abstract}
New benztriazoles with a mercapto-triazole and other heterocycle substituents were synthesized and evaluated for their anticonvulsant activity and neurotoxicity by using the maximal electroshock (MES), subcutaneous pentylenetetrazole (scPTZ), and rotarod neurotoxicity (TOX) tests. Among the compounds studied, compound 2-((1H-1,2,4-triazol-3-yl)thio)-N-(6-((3-fluorobenzyl)

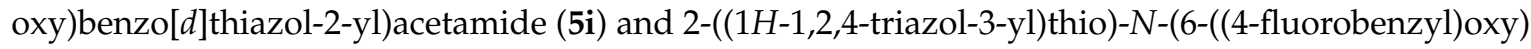
benzo[d] thiazol-2-yl)acetmide (5j) were the most potent, with an $\mathrm{ED}_{50}$ value of $50.8 \mathrm{mg} / \mathrm{kg}$ and $54.8 \mathrm{mg} / \mathrm{kg}$ in the MES test and $76.0 \mathrm{mg} / \mathrm{kg}$ and $52.8 \mathrm{mg} / \mathrm{kg}$ in the scPTZ seizures test, respectively. They also showed lower neurotoxicity and, therefore a higher protective index. In particular, compound $5 \mathbf{j}$ showed high protective index (PI) values of 8.96 in the MES test and 9.30 in the scPTZ test, which were better than those of the standard drugs used as positive controls in this study.
\end{abstract}

Keywords: synthesis; benzothiazole; mercapto-triazole; anticonvulsant; maximal electroshock; neurotoxicity; pentylenetetrazole

\section{Introduction}

February ninth, 2015 was the first International Epilepsy Day, and it helped more people raise awareness and understanding about epilepsy. Such efforts are urgently needed, because with more than 50 million people presenting with epilepsy worldwide, epilepsy is the most common, chronic, serious neurological disease. In 2013, 119,000 deaths worldwide were attributable to epilepsy. Currently, $40 \%$ of patients in high-income countries and more than $70 \%$ of patients in developing countries do not get the treatment they need, because of the high expense or low availability of the appropriate drugs [1-3]. Therefore, there is a pressing need to develop more effective antiepileptic drugs (AEDs) endowed with an improved safety profile.

On the basis of a number of related materials, azoles and their derivatives have gained much attention in recent years due to their potential biological applications linked to their anticonvulsant [4], anti-inflammatory [5], anti-fungal [6], antiviral [7], and anticancer [8] activities. Among them, heterocyclic compounds with the 3-mercapto-1,2,4-triazole substructure exhibit a wide spectrum of biological activities $[9,10]$. Similarly, we also demonstrated that the benzothiazole nucleus is a unique scaffold for further molecular exploration to synthesize novel compounds. A literature survey revealed that benzothiazole analogs are associated with diverse pharmacological effects [11-14], including anticonvulsant activity [15,16]. For this reason, and in continuation to our efforts directed toward the synthesis of new heterocyclic compounds with 
anticonvulsant biological activities, in this study, we combined both biological components (3-mercapto-1,2,4-triazole and benzothiazole) with an amide, which has anticonvulsant bioactivities in some AEDs (such as carbamazepine and riluzole, also see references $[17,18]$ ), to obtain a series of 2-((1H-1,2,4-triazol-3-yl)thio)-N-(6-alkoxybenzo[d]thiazol-2-yl)acetamide. To compare the compound activities with those of other azoles, the mercapto-triazole in compound 5 was replaced with other heterocycles such as imidazole, triazole, tetrazole, and 3-amino-1,2,4-triazole, to obtain compounds 6, 7, 8, and 9 (Figure 1). Their anticonvulsant activities were evaluated using the maximal electroshock (MES) test and their neurotoxicity (TOX) was evaluated with the rotarod test in mice.
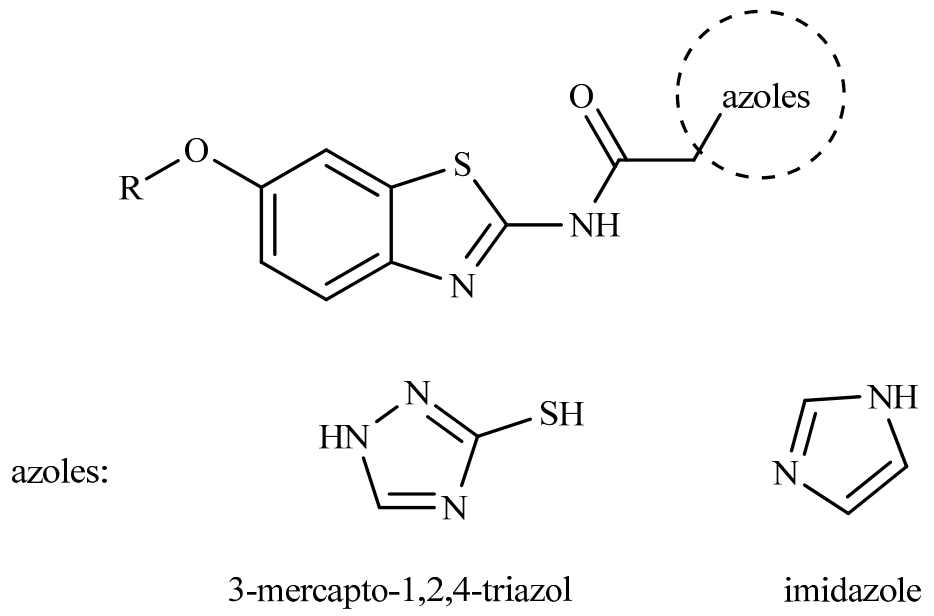

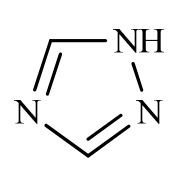

1,2,4-triazol<smiles>c1nnn[nH]1</smiles>

tetrazole<smiles>Nc1nc[nH]n1</smiles>

3-amino-1,2,4-triazole

Figure 1. Benzothiazole and kinds of azoles combined with amide.

\section{Results and Discussion}

\subsection{Chemistry}

All the target compounds were synthesized according to Scheme 1. Compounds $\mathbf{3 a - m}$ were prepared according to previous studies in our laboratory [19]. Compounds 3a-m were treated with chloroacetyl chloride at room temperature in acetone to yield derivatives $\mathbf{4 a}-\mathbf{m}[20,21]$. Finally, when derivatives $\mathbf{4 a - m}$ were allowed to react with different azoles, such as 1H-1,2,4-triazole-3-thiol, imidazole, triazole, tetrazole, and 3-amino-1,2,4-triazole in refluxing dimethylformamide (DMF) in the presence of $\mathrm{NaOH}$ [22-25], the 2-chlorine atom was substituted by these heterocycles, producing the corresponding compounds: 2-((1H-1,2,4-triazol-3-yl)thio)-N-(6-alkoxybenzo[d]thiazol-2-yl)acetamide (5a-m), $N$-(6-alkoxybenzo[d]thiazol-2-yl)-2-(1H-imidazol-1-yl)acetamide (6a-b), $N$-(6-alkoxybenzo[d] thiazol-2-yl)-2-(1H-1,2,4-triazol-1-yl)acetamide (7a-b), N-(6-alkoxybenzo[d]thiazol-2-yl)-2-(1H-tetrazol-1-yl) acetamide (8a-b), and 2-(3-amino-1H-1,2,4-triazol-1-yl)-N-(6-alkoxybenzo[d] thiazol-2-yl) acetamide $(\mathbf{9 a}-\mathbf{b})$. The structures of the targeted compounds were characterized using spectral methods, and all spectral data corroborated the assumed structures. 


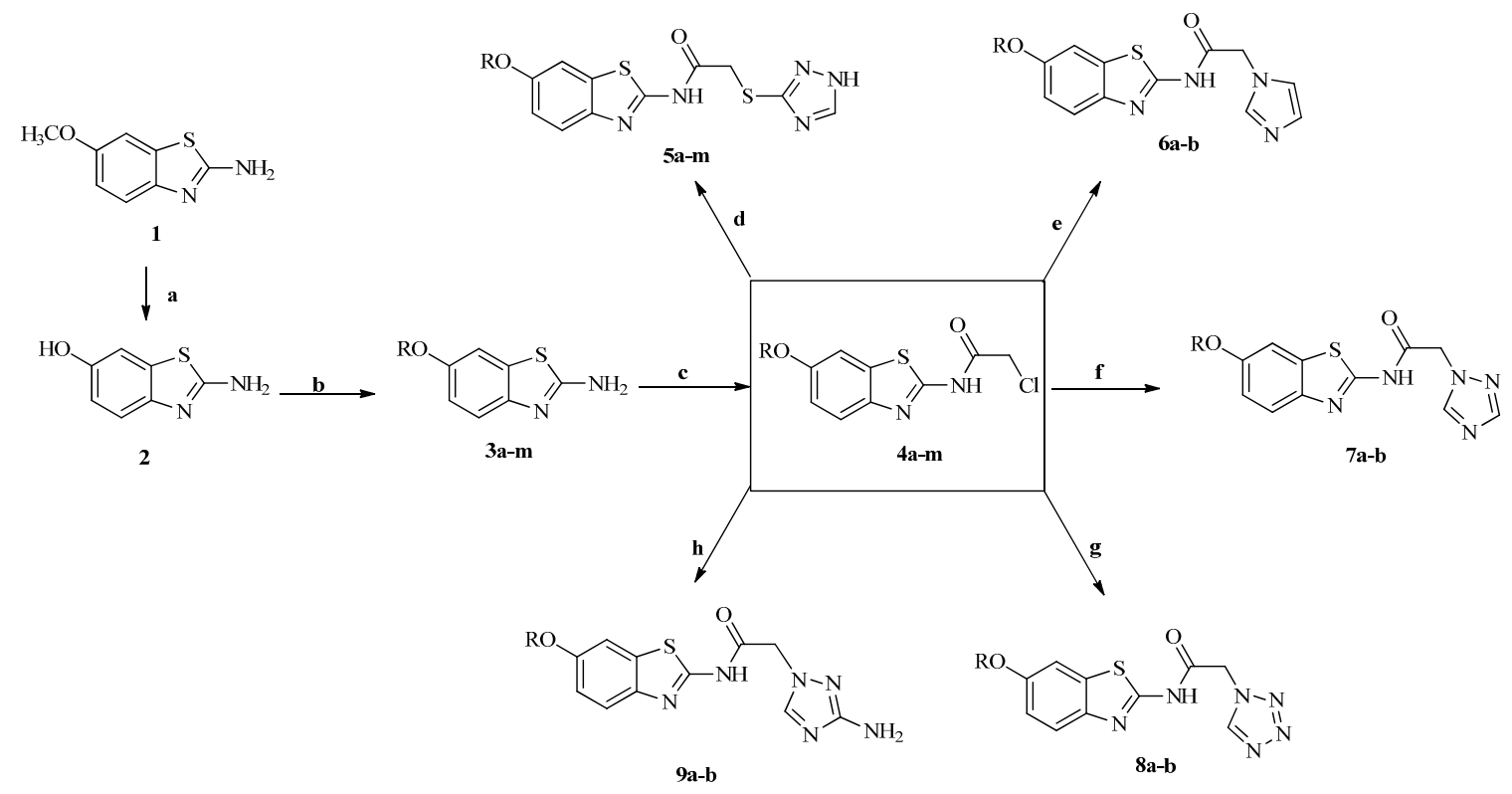

Scheme 1. Synthetic route of target compounds. Reagents and conditions: (a) $\mathrm{HBr}(48 \mathrm{wt} \%$ in $\mathrm{H}_{2} \mathrm{O}, 99.99 \%$ metals basis), reflux, $18 \mathrm{~h}$; (b) $\mathrm{RBr} / \mathrm{RPHCH}_{2} \mathrm{Cl}, \mathrm{CH}_{3} \mathrm{COCH}_{3}, \mathrm{~K}_{2} \mathrm{CO}_{3}$, reflux, 18-24 h;

(c) $\mathrm{ClCOCH}_{2} \mathrm{Cl}$, acetone, room temperature, 9-10 h; (d-h) DMF, $\mathrm{NaOH}$, room temperature, 8-12 h.

\subsection{Pharmacology and Structure-Activity Relationship}

The anticonvulsant activity evaluation of compounds 2-((1H-1,2,4-triazol-3-yl) thio)- $N$ -(6-alkoxybenzo[d]thiazol-2-yl)acetamide (5a-m) were determined using the MES test, which is a mechanism-independent animal seizure model that enables the identification of compounds preventing seizure spread [26]. It should be noted that the MES model remains the most useful tool for the identification of new anticonvulsants, despite significant advances in epilepsy research in the past several years [27]. The MES seizure model was used for preliminary (phase I) screening of compounds $\mathbf{5 a}-\mathbf{m}$. They were administered to mice intraperitoneally (i.p.) at the fixed dose of $100 \mathrm{mg} / \mathrm{kg}$ and the anticonvulsant protection was observed at two post-treatment times: 0.5 and $4 \mathrm{~h}$. The method applied here allowed the determination of the number of animals (in a group consisting of three mice) protected against electrically-induced seizures as well as the estimation of the time course of anticonvulsant activity, including quick-acting $(0.5 \mathrm{~h})$ or long-acting properties $(4 \mathrm{~h})$. The results are presented in Table 1 . The preliminary pharmacological screening revealed that five compounds $(\mathbf{5 b}, \mathbf{5} \mathbf{c}, \mathbf{5} \mathbf{g}, \mathbf{5 i}$, and $5 \mathbf{j}$ ) showed $100 \%$ anticonvulsant protection in the $0.5 \mathrm{~h}$ period and some of them $(\mathbf{5 c}, \mathbf{5 i}$, and $\mathbf{5 j})$ still had a little activity in the $4 \mathrm{~h}$ period. One compound, $5 \mathrm{~h}$, showed $67 \%$ anticonvulsant protection in the $0.5 \mathrm{~h}$ period, but no activity in the $4 \mathrm{~h}$ period. None of the compounds presented neurotoxicity at the dose of $100 \mathrm{mg} / \mathrm{kg}$. Based on the above preliminary data, six active compounds were screened at the dose of $30 \mathrm{mg} / \mathrm{kg}$ in mice (i.p.) at the two post-treatment times $(0.5 \mathrm{~h}$ and $4 \mathrm{~h})$. As shown in Table 2, only two compounds, $5 \mathbf{i}$ and $5 \mathbf{j}$, showed about $33 \%$ anticonvulsant protection activity in the $0.5 \mathrm{~h}$ period but no activity in the $4 \mathrm{~h}$ period.

The following structure-activity relationships (SAR) were obtained, while analyzing the preliminary screening of the synthesized compounds. Among the six alkyl chain-substituted derivatives, $\mathbf{5 b}$ and $\mathbf{5 c}$ showed better activities, and $\mathbf{5 c}$ still presented some activities in the $4 \mathrm{~h}$ period. However, with the increase in length, the activities of the compounds did not increase. Compound 5g, substituted with a benzyl group at the 6-position of the benzothiazole core, showed moderate activity at $100 \mathrm{mg} / \mathrm{kg}$. Thus, the $\mathrm{F}, \mathrm{Cl}$, and $\mathrm{CF}_{3}$ groups were subsequently added onto the benzyloxy group of $\mathbf{5 g}$ at different positions, yielding compounds $\mathbf{5 h}-\mathbf{m}$. Substituent position on the phenyl ring also influenced anticonvulsant activity in the 6-fluorobenzyl derivatives as $m-\mathrm{F}=p-\mathrm{F}>0$ - . However, the 6-chlorobenzyl derivatives showed no activity at the dose of $100 \mathrm{mg} / \mathrm{kg}$. Compound $5 \mathrm{~m}$, 
substituted with a trifluoromethyl at the 3-position of the benzyl group, also showed no activity, regardless of the period $(0.5 \mathrm{~h}$ or $4 \mathrm{~h})$ at the dose of $100 \mathrm{mg} / \mathrm{kg}$. Based on the above-mentioned results, six compounds $(\mathbf{5 b}, \mathbf{5 c}, \mathbf{5 g}, \mathbf{5 h}, \mathbf{5 i}$, and $\mathbf{5 j})$ were selected from all the compounds for the next step and were tested at a dose of $30 \mathrm{mg} / \mathrm{kg}$. As shown in Table 2, at the dose of $30 \mathrm{mg} / \mathrm{kg}$, only $m-\mathrm{F}$ and $p$-F substituted compounds presented some anticonvulsant activities in the $0.5 \mathrm{~h}$ period.

Table 1. Anticonvulsant activities screening (maximal electroshock test) and neurotoxicity screening in mice at the dose of $100 \mathrm{mg} / \mathrm{kg}$.

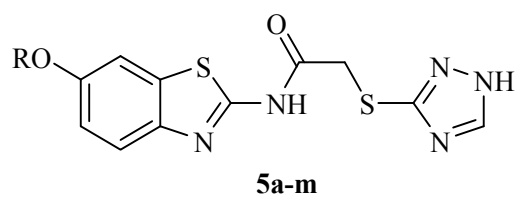

\begin{tabular}{|c|c|c|c|c|c|}
\hline \multirow{2}{*}{ Comp. } & \multirow{2}{*}{$\mathbf{R}$} & \multicolumn{2}{|c|}{$\operatorname{MES}(100 \mathrm{mg} / \mathrm{kg})^{a}$} & \multicolumn{2}{|c|}{ Toxicity $(100 \mathrm{mg} / \mathrm{kg})$} \\
\hline & & $0.5 \mathrm{~h}$ & $4 \mathrm{~h}$ & $0.5 \mathrm{~h}$ & $4 \mathrm{~h}$ \\
\hline $5 a$ & $-\mathrm{CH}_{3}$ & $1 / 3^{b}$ & $0 / 3$ & $0 / 3$ & $0 / 3$ \\
\hline $5 b$ & $n-\mathrm{C}_{3} \mathrm{H}_{7}$ & $3 / 3$ & $0 / 3$ & $0 / 3$ & $0 / 3$ \\
\hline $5 c$ & $n-\mathrm{C}_{4} \mathrm{H}_{9}$ & $3 / 3$ & $1 / 3$ & $0 / 3$ & $0 / 3$ \\
\hline $5 d$ & $n-\mathrm{C}_{5} \mathrm{H}_{11}$ & $0 / 3$ & $0 / 3$ & $0 / 3$ & $0 / 3$ \\
\hline $5 e$ & $n-\mathrm{C}_{6} \mathrm{H}_{13}$ & $1 / 3$ & $0 / 3$ & $0 / 3$ & $0 / 3$ \\
\hline $5 f$ & $n-\mathrm{C}_{7} \mathrm{H}_{15}$ & $1 / 3$ & $0 / 3$ & $0 / 3$ & $0 / 3$ \\
\hline $5 g$ & $-\mathrm{CH}_{2} \mathrm{C}_{6} \mathrm{H}_{5}$ & $3 / 3$ & $0 / 3$ & $0 / 3$ & $0 / 3$ \\
\hline $5 \mathrm{~h}$ & $-\mathrm{CH}_{2} \mathrm{C}_{6} \mathrm{H}_{4}(o-\mathrm{F})$ & $2 / 3$ & $0 / 3$ & $0 / 3$ & $0 / 3$ \\
\hline $5 i$ & $-\mathrm{CH}_{2} \mathrm{C}_{6} \mathrm{H}_{4}(m-\mathrm{F})$ & $3 / 3$ & $1 / 3$ & $0 / 3$ & $0 / 3$ \\
\hline $5 \mathbf{j}$ & $-\mathrm{CH}_{2} \mathrm{C}_{6} \mathrm{H}_{4}(p-\mathrm{F})$ & $3 / 3$ & $1 / 3$ & $0 / 3$ & $0 / 3$ \\
\hline $5 k$ & $-\mathrm{CH}_{2} \mathrm{C}_{6} \mathrm{H}_{4}(o-\mathrm{Cl})$ & $0 / 3$ & $0 / 3$ & $0 / 3$ & $0 / 3$ \\
\hline 51 & $-\mathrm{CH}_{2} \mathrm{C}_{6} \mathrm{H}_{4}(m-\mathrm{Cl})$ & $0 / 3$ & $0 / 3$ & $0 / 3$ & $0 / 3$ \\
\hline $5 \mathrm{~m}$ & $-\mathrm{CH}_{2} \mathrm{C}_{6} \mathrm{H}_{4}\left(m-\mathrm{CF}_{3}\right)$ & $0 / 3$ & $0 / 3$ & $0 / 3$ & $0 / 3$ \\
\hline
\end{tabular}

a Maximal electroshock (MES): doses of $100 \mathrm{mg} / \mathrm{kg}$ were administrated intraperitoneally in mice. The animals were examined at two times: $0.5 \mathrm{~h}$ and $4 \mathrm{~h}$ after administration; ${ }^{\mathrm{b}} \mathrm{n} 1 / \mathrm{n} 2$ : the animals protected/the animals tested.

Table 2. Anticonvulsant activities screening: MES test in mice at the dose of $30 \mathrm{mg} / \mathrm{kg}$.

\begin{tabular}{|c|c|c|c|}
\hline \multirow{2}{*}{ Comp. } & \multirow{2}{*}{$\mathbf{R}$} & \multicolumn{2}{|c|}{$\operatorname{MES}(30 \mathrm{mg} / \mathrm{kg})^{a}$} \\
\hline & & $0.5 \mathrm{~h}$ & $4 \mathrm{~h}$ \\
\hline $5 b$ & $n-\mathrm{C}_{3} \mathrm{H}_{7}$ & $0 / 3$ & $0 / 3$ \\
\hline $5 c$ & $n-\mathrm{C}_{4} \mathrm{H}_{9}$ & $0 / 3$ & $0 / 3$ \\
\hline $5 g$ & $-\mathrm{CH}_{2} \mathrm{C}_{6} \mathrm{H}_{5}$ & $0 / 3$ & $0 / 3$ \\
\hline $5 \mathrm{~h}$ & $-\mathrm{CH}_{2} \mathrm{C}_{6} \mathrm{H}_{4}(o-\mathrm{F})$ & $0 / 3$ & $0 / 3$ \\
\hline $5 i$ & $-\mathrm{CH}_{2} \mathrm{C}_{6} \mathrm{H}_{4}(m-\mathrm{F})$ & $1 / 3$ & $0 / 3$ \\
\hline $5 j$ & $-\mathrm{CH}_{2} \mathrm{C}_{6} \mathrm{H}_{4}(p-\mathrm{F})$ & $1 / 3$ & $0 / 3$ \\
\hline
\end{tabular}

a Maximal electroshock (MES): doses of $30 \mathrm{mg} / \mathrm{kg}$ were administrated intraperitoneally in mice.

According to the bioisosterism, the mercapto-triazole ring of compounds $5 \mathbf{i}$ and $5 \mathbf{j}$ was replaced with other heterocycles, such as imidazole, triazole, tetrazole, and 3-amino-1,2,4-triazole. Compounds $6,7,8$, and 9 were designed and synthesized. Their anticonvulsant activities were evaluated at the dose of $100 \mathrm{mg} / \mathrm{kg}$, and screening results are shown in Table 3. The compounds, substituted with other heterocycles, hardly showed any anticonvulsant activities in the $0.5 \mathrm{~h}$ or $4 \mathrm{~h}$ period. Thus, we concluded that when the mercapto-triazole ring in compounds $\mathbf{5 a}-\mathbf{m}$ was replaced by other heterocycles (i.e., imidazole, triazole, tetrazole, and 3-amino-1,2,4-triazole), the resultant compounds, $\mathbf{6 a}-\mathbf{b}, \mathbf{7 a}-\mathbf{b}, \mathbf{8} \mathbf{a}-\mathbf{b}$, and $\mathbf{9 a}-\mathbf{b}$, hardly presented any activities compared with the compounds containing the mercapto-triazole ring. 
Table 3. Anticonvulsant activities of compounds 6, 7, 8, and 9 in MES test.<smiles>[R20]c1ccc2nc(NC(=O)Cn3ccnc3)sc2c1</smiles><smiles>O=C(Cn1cncn1)Nc1nc2ccc(O[Ga])cc2s1</smiles><smiles>[R]Oc1ccc2nc(NC(=O)Cn3cnnn3)sc2c1</smiles><smiles>[R20]c1ccc2nc(NC(=O)Cn3cnc(N)n3)sc2c1</smiles>

9a-b

\begin{tabular}{|c|c|c|c|c|c|}
\hline \multirow{2}{*}{ Comp. } & \multirow{2}{*}{$\mathbf{R}$} & \multicolumn{2}{|c|}{ MES $(100 \mathrm{mg} / \mathrm{kg})$} & \multicolumn{2}{|c|}{ Toxicity $(100 \mathrm{mg} / \mathrm{kg})$} \\
\hline & & $0.5 \mathrm{~h}$ & $4 \mathrm{~h}$ & $0.5 \mathrm{~h}$ & $4 \mathrm{~h}$ \\
\hline $6 a$ & $-\mathrm{CH}_{2} \mathrm{C}_{6} \mathrm{H}_{4}(m-\mathrm{F})$ & $0 / 3$ & $0 / 3$ & $0 / 3$ & $0 / 3$ \\
\hline $6 b$ & $-\mathrm{CH}_{2} \mathrm{C}_{6} \mathrm{H}_{4}(p-\mathrm{F})$ & $0 / 3$ & $0 / 3$ & $0 / 3$ & $0 / 3$ \\
\hline $7 a$ & $-\mathrm{CH}_{2} \mathrm{C}_{6} \mathrm{H}_{4}(m-\mathrm{F})$ & $0 / 3$ & $0 / 3$ & $0 / 3$ & $0 / 3$ \\
\hline $7 b$ & $-\mathrm{CH}_{2} \mathrm{C}_{6} \mathrm{H}_{4}(p-\mathrm{F})$ & $0 / 3$ & $0 / 3$ & $0 / 3$ & $0 / 3$ \\
\hline $8 a$ & $-\mathrm{CH}_{2} \mathrm{C}_{6} \mathrm{H}_{4}(m-\mathrm{F})$ & $0 / 3$ & $0 / 3$ & $0 / 3$ & $0 / 3$ \\
\hline $8 b$ & $-\mathrm{CH}_{2} \mathrm{C}_{6} \mathrm{H}_{4}(p-\mathrm{F})$ & $0 / 3$ & $0 / 3$ & $0 / 3$ & $0 / 3$ \\
\hline $9 a$ & $-\mathrm{CH}_{2} \mathrm{C}_{6} \mathrm{H}_{4}(m-\mathrm{F})$ & $1 / 3$ & $1 / 3$ & $0 / 3$ & $0 / 3$ \\
\hline $9 b$ & $-\mathrm{CH}_{2} \mathrm{C}_{6} \mathrm{H}_{4}(p-\mathrm{F})$ & $0 / 3$ & $0 / 3$ & $0 / 3$ & $0 / 3$ \\
\hline
\end{tabular}

On the basis of the preliminary screening results, compounds $5 \mathbf{i}$ and $5 \mathbf{j}$ were subjected to the next phase of trials regarding the quantification of their anticonvulsant activity (indicated by $\mathrm{ED}_{50} \mathrm{MES}$ ) in mice.

The MES and subcutaneous pentylenetetrazole (scPTZ) seizure models represent the two most widely used animal seizure models in the search for new AEDs. The scPTZ test employs chemically-induced myoclonic seizures and allows the identification of agents raising the seizure threshold. This test is related to human generalized absence seizures [28]. Thus, the quantitative analysis of compounds $5 \mathbf{i}$ and $5 \mathbf{j}$, the most potent compounds in the MES test, was studied in the scPTZ test to obtain $\mathrm{ED}_{50}$ PTZ. The quantitative neurotoxicity data (indicated by $\mathrm{TD}_{50}$ ) of compounds $5 \mathbf{i}$ and $5 \mathbf{j}$ was obtained from the rotarod test.

The results of the quantitative tests are reported in Table 4, along with the data from carbamazepine and phenytoin as positive drug control groups. The quantitative i.p. data in mice confirmed the safe and potent anticonvulsant activity of $5 \mathbf{i}$ and $5 \mathbf{j}$. As shown in Table 4, both compounds showed a weaker anticonvulsant activity than the control drug carbamazepine (the ED $\mathrm{D}_{50}$ MES was $11.8 \mathrm{mg} / \mathrm{kg}$ ) in the MES seizure model. However, they showed a stronger anticonvulsant activity than valproic acid (the ED 50 MES was $216.9 \mathrm{mg} / \mathrm{kg}$ ) and better activities in the scPTZ test than all the drugs used as positive controls in this study. Especially, compound 5j showed higher safety with lower neurotoxicity than compound 5i, resulting in higher PI values (8.96 in the MES test and 9.30 in the scPTZ test).

Table 4. Quantitative Pharmacological Parameters $\mathrm{ED}_{50}, \mathrm{TD}_{50}$, and PI Values in Mice.

\begin{tabular}{|c|c|c|c|c|c|}
\hline \multirow{2}{*}{ Comp. } & \multirow{2}{*}{$\mathrm{ED}_{50}{ }^{\mathrm{a}} \mathrm{MES}(\mathrm{mg} / \mathrm{kg})$} & \multirow{2}{*}{$\mathrm{ED}_{50} \mathrm{scPTZ}^{\mathrm{b}}(\mathrm{mg} / \mathrm{kg})$} & \multirow{2}{*}{$\mathrm{TD}_{50}{ }^{\mathrm{c}}(\mathrm{mg} / \mathrm{kg})$} & \multicolumn{2}{|c|}{ PI $^{d}$} \\
\hline & & & & MES & scPTZ \\
\hline $5 i$ & $50.8(37.0-69.8)^{\mathrm{e}}$ & $76.0(65.9-87.7)$ & $353.5(309.3-404.1)$ & 6.96 & 4.65 \\
\hline $5 \mathbf{j}$ & $54.8(36.7-81.8)$ & $52.8(45.8-60.9)$ & $491.0(429.5-561.2)$ & 8.96 & 9.30 \\
\hline CBZ & $11.8(8.5-16.4)$ & $>100$ & $76.1(55.8-103.7)$ & 6.45 & $<0.76$ \\
\hline VPA & $216.9(207.5-226.3)$ & $239.4(209.2-274.1)$ & $372.9(356.0-389.8)$ & 1.72 & 1.56 \\
\hline
\end{tabular}

a $\mathrm{ED}_{50}$ : median effective dose affording anticonvulsant protection in $50 \%$ of animals; ${ }^{\mathrm{b}}$ scPTZ: subcutaneous pentylenetetrazole seizure test; ${ }^{\mathrm{c}} \mathrm{TD}_{50}$ : median toxic dose eliciting minimal neurological toxicity in $50 \%$ of animals; ${ }^{\mathrm{d}} \mathrm{PI}$ : protective index $\left(\mathrm{TD}_{50} / \mathrm{ED}_{50}\right) ;{ }^{\mathrm{e}} 95 \%$ confidence intervals given in parentheses. 


\section{Experimental Procedures}

\subsection{General Information}

Melting points were determined in open capillary tubes and were uncorrected. IR spectra were recorded (in $\mathrm{KBr}$ ) on IR Prestige-21. ${ }^{1} \mathrm{H}-\mathrm{NMR}$ and ${ }^{13} \mathrm{C}-\mathrm{NMR}$ spectra were measured on an AV-300 (Bruker, Switzerland), and all chemical shifts were given in ppm relative to tetramethylsilane. Mass spectra were measured on an AXIMA CFR Plus MALDI-TOF (Shimadzu, Japan). The chemicals were purchased from Aldrich Chemical Corporation.

\subsection{Chemistry}

\subsubsection{Synthesis of 6-Hydroxy-2-aminobenzothiazole (2)}

A mixture of 6-methoxy-2,3-dihydrobenzo[d]thiazol-2-amine (1) $(10 \mathrm{~g}, 55.56 \mathrm{mmol})$ and $40 \mathrm{~mL}$ of hydrobromic acid (48\% water solution) was refluxed for $20 \mathrm{~h}$. The mixture was allowed to cool to room temperature and neutralized with $\mathrm{NaOH}$ solution to $\mathrm{pH} 7-8$. The precipitate was filtered and washed with water. The filtrate was stirred with $100 \mathrm{~mL}$ hot water for $0.5 \mathrm{~h}$ and the remaining precipitate was filtered to yield a brown solid, compound 2 .

\subsubsection{General Procedure for the Synthesis of 6-Alkoxy-2-aminobenzothiazoles (3a-m)}

A mixture of compound $2(2 \mathrm{~g}, 12 \mathrm{mmol})$, potassium carbonate $(2 \mathrm{~g}, 14.4 \mathrm{mmol})$, appropriate alkyl bromide or benzyl chloride derivatives $(1.32 \mathrm{mmol})$, and a catalytic amount of benzyltriethylamine chloride (TEBA) in $50 \mathrm{~mL}$ acetone was heated under reflux for 18-24 h. After removing the solvent under reduced pressure, $80 \mathrm{~mL}$ of hot water was poured into the flask and the mixture was stirred for $0.5 \mathrm{~h}$ to eliminate the excess of potassium carbonate. The remaining precipitate was filtered to yield a russet solid (3a-m), which was used without further purification.

3.2.3. General Procedure for the Synthesis of 2-Chloro- $N$-(6-alkoxybenzo[d]thiazol-2-yl)acetamide $(4 \mathrm{a}-\mathrm{m})$

6-Alkoxy-2-aminobenzothiazoles (3a-m) (20 mmol) was dissolved in $30 \mathrm{~mL}$ acetone and $30 \mathrm{mmol}$ of chloroacetyl chloride were added under cold conditions. The reaction was stirred for 9-10 h at room temperature. Water was added to the reaction mixture after the solvent (acetone) was removed under pressure-reducing conditions. The mixture was stirred for $0.5 \mathrm{~h}$ and filtered to yield a crude product. It was then purified by silica gel column chromatography with dichloromethane to yield a white solid $\mathbf{4 a}-\mathbf{m}$.

3.2.4. General Procedure for the Synthesis of 2-((1H-1,2,4-Triazol-3-yl)thio)-N-(6-alkoxybenzo[d] thiazol-2-yl)acetamide (5a-m)

A mixture of 2-chloro- $N$-(6-alkoxybenzo[d]thiazol-2-yl)acetamide (4a-m) (5 mmol), 1,2,4-triazol-3-thiol (6 mmol), and sodium hydroxide $(6 \mathrm{mmol})$ in $N, N$-dimethylformamide $(10 \mathrm{~mL})$ was stirred at room temperature for $12 \mathrm{~h}$. After removing half of the solvent, $50 \mathrm{~mL}$ of water was added to the reaction mixture, which was then extracted with ethyl acetate $(30 \mathrm{~mL} \times 3)$. The combined layer of ethyl acetate was dried over anhydrous $\mathrm{MgSO}_{4}$. Evaporation of the solvent provided a crude product, which was recrystallized from dichloromethane to yield a white solid.

The yield, melting point, analytical data and spectral data of each compound are given below.

2-((1H-1,2,4-Triazol-3-yl)thio)-N-(6-methoxybenzo[d]thiazol-2-yl)acetamide (5a). White solid in 52.5\%. mp: 208-209 ${ }^{\circ} \mathrm{C} .{ }^{1} \mathrm{H}-\mathrm{NMR}(300 \mathrm{MHz}, \mathrm{DMSO}) \delta 14.02$ (s, 1H, triazole -NH-), 12.57 (s, 1H, -CO-NH-), 8.50 $(\mathrm{s}, 1 \mathrm{H}$, triazole $=\mathrm{CH}-), 7.65(\mathrm{~d}, J=8.8 \mathrm{~Hz}, 1 \mathrm{H}, \mathrm{Ar}-\mathrm{H}), 7.58(\mathrm{~d}, J=2.5 \mathrm{~Hz}, 1 \mathrm{H}, \mathrm{Ar}-\mathrm{H}), 7.03(\mathrm{dd}, J=8.8$, $2.6 \mathrm{~Hz}, 1 \mathrm{H}, \mathrm{Ar}-\mathrm{H}), 4.20$ (s, 2H, $\left.-\mathrm{S}-\mathrm{CH}_{2}-\right)$, $3.80\left(\mathrm{~s}, 3 \mathrm{H},-\mathrm{OCH}_{3}\right) .{ }^{13} \mathrm{C}-\mathrm{NMR}(75 \mathrm{MHz}, \mathrm{DMSO}) \delta 167.44$, 
156.18, 155.77, 145.31, 144.89, 142.60, 132.79, 121.21, 114.97, 104.71, 55.61, 35.07. IR (KBr) cm ${ }^{-1}: 1605.02$, $1556.65(\mathrm{C}=\mathrm{N})$. Tof-MS: $m / z[\mathrm{M}+\mathrm{H}]^{+} 322.26$.

2-((1H-1,2,4-Triazol-3-yl)thio)-N-(6-propoxybenzo[d]thiazol-2-yl)acetamide (5b). White solid in $65.9 \%$. mp: 169-170 ${ }^{\circ} \mathrm{C} .{ }^{1} \mathrm{H}-\mathrm{NMR}(300 \mathrm{MHz}, \mathrm{DMSO}) \delta 14.09$ (s, 1H, triazole -NH-), 12.50 (s, 1H, -CO-NH-), 8.51 $(\mathrm{s}, 1 \mathrm{H}$, triazole $=\mathrm{CH}-), 7.64(\mathrm{~d}, J=8.8 \mathrm{~Hz}, 1 \mathrm{H}, \mathrm{Ar}-\mathrm{H}), 7.55(\mathrm{~s}, 1 \mathrm{H}, \mathrm{Ar}-\mathrm{H}), 7.03(\mathrm{dd}, J=8.8,2.5 \mathrm{~Hz}, 1 \mathrm{H}$, $\mathrm{Ar}-\mathrm{H}), 4.19\left(\mathrm{~s}, 2 \mathrm{H},-\mathrm{S}-\mathrm{CH}_{2}-\right), 3.97\left(\mathrm{t}, J=6.4 \mathrm{~Hz}, 2 \mathrm{H},-\mathrm{OCH}_{2}-\right), 1.75\left(\mathrm{dd}, J=13.9,6.8 \mathrm{~Hz}, 2 \mathrm{H},-\mathrm{CH}_{2}-\right)$, $0.99\left(\mathrm{t}, J=7.3 \mathrm{~Hz}, 3 \mathrm{H},-\mathrm{CH}_{3}\right) .{ }^{13} \mathrm{C}-\mathrm{NMR}(75 \mathrm{MHz}, \mathrm{DMSO}) \delta 167.41,155.73,155.57,145.55,145.23,142.52$, 132.77, 121.19, 115.33, 105.41, 69.54, 35.04, 22.09, 10.43. IR (KBr) $\mathrm{cm}^{-1}: 1605.75,1550.35$ (C=N). Tof-MS: $m / z[\mathrm{M}+\mathrm{H}]^{+} 350.15$.

2-((1H-1,2,4-Triazol-3-yl)thio)-N-(6-butoxybenzo[d]thiazol-2-yl)acetamide (5c). White solid in 40.6\%. mp: 172-173 ${ }^{\circ} \mathrm{C} .{ }^{1} \mathrm{H}-\mathrm{NMR}(300 \mathrm{MHz}, \mathrm{DMSO}) \delta 14.11(\mathrm{~s}, 1 \mathrm{H}$, triazole $-\mathrm{NH}-), 12.45(\mathrm{~s}, 1 \mathrm{H},-\mathrm{CO}-\mathrm{NH}-), 8.58$ $(\mathrm{s}, 1 \mathrm{H}$, triazole $=\mathrm{CH}-), 7.63(\mathrm{~d}, J=8.8 \mathrm{~Hz}, 1 \mathrm{H}, \mathrm{Ar}-\mathrm{H}), 7.55(\mathrm{~s}, 1 \mathrm{H}, \mathrm{Ar}-\mathrm{H}), 7.02(\mathrm{dd}, J=8.8,2.5 \mathrm{~Hz}, 1 \mathrm{H}$, $\mathrm{Ar}-\mathrm{H}), 4.18\left(\mathrm{~s}, 2 \mathrm{H},-\mathrm{S}-\mathrm{CH}_{2}-\right), 4.01\left(\mathrm{t}, J=6.4 \mathrm{~Hz}, 2 \mathrm{H},-\mathrm{OCH}_{2}-\right), 1.80-1.64\left(\mathrm{~m}, 2 \mathrm{H},-\mathrm{CH}_{2}-\right), 1.54-1.36$ $\left(\mathrm{m}, 2 \mathrm{H},-\mathrm{CH}_{2}-\right), 0.94\left(\mathrm{t}, J=7.3 \mathrm{~Hz}, 3 \mathrm{H},-\mathrm{CH}_{3}\right) .{ }^{13} \mathrm{C}-\mathrm{NMR}(75 \mathrm{MHz}, \mathrm{DMSO}) \delta 167.39,155.73,155.64$, $145.41,145.13,142.57,132.81,121.18,115.35,105.50,67.84,35.12,30.81,18.77,13.67$. IR (KBr) cm ${ }^{-1}$ : $1605.78,1556.09(\mathrm{C}=\mathrm{N})$. Tof-MS: $m / z[\mathrm{M}+\mathrm{H}]^{+} 363.74$.

2-((1H-1,2,4-Triazol-3-yl)thio)-N-(6-(pentyloxy)benzo[d] thiazol-2-yl)acetamide (5d). White solid in 37.2\%. mp: 107-108 ${ }^{\circ} \mathrm{C} .{ }^{1} \mathrm{H}-\mathrm{NMR}(300 \mathrm{MHz}, \mathrm{DMSO}) \delta 14.07$ (s, 1H, triazole -NH-), 12.46 (s, 1H, -CO-NH-), $8.51(\mathrm{~s}, 1 \mathrm{H}$, triazole $=\mathrm{CH}-), 7.63(\mathrm{~d}, J=8.8 \mathrm{~Hz}, 1 \mathrm{H}, \mathrm{Ar}-\mathrm{H}), 7.56(\mathrm{~s}, 1 \mathrm{H}, \mathrm{Ar}-\mathrm{H}), 7.02(\mathrm{dd}, J=8.8,2.5 \mathrm{~Hz}$, $1 \mathrm{H}, \mathrm{Ar}-\mathrm{H}), 4.19\left(\mathrm{~s}, 2 \mathrm{H},-\mathrm{S}-\mathrm{CH}_{2}-\right), 4.00\left(\mathrm{t}, J=6.5 \mathrm{~Hz}, 2 \mathrm{H},-\mathrm{OCH}_{2}-\right), 1.84-1.64\left(\mathrm{~m}, 2 \mathrm{H},-\mathrm{CH}_{2}-\right), 1.39$ $\left(\mathrm{dd}, J=15.1,10.5 \mathrm{~Hz}, 4 \mathrm{H},-\mathrm{CH}_{2}-\right), 0.90\left(\mathrm{t}, J=7.0 \mathrm{~Hz}, 3 \mathrm{H},-\mathrm{CH}_{3}\right) .{ }^{13} \mathrm{C}-\mathrm{NMR}(75 \mathrm{MHz}, \mathrm{DMSO}) \delta 167.41$, 155.72, 155.59, 145.60, 145.26, 142.51, 132.77, 121.18, 115.32, 105.38, 68.03, 35.05, 28.43, 27.75, 21.91, 13.92. IR (KBr) cm ${ }^{-1}:$ 1606.70, $1550.71(\mathrm{C}=\mathrm{N})$. Tof-MS: $m / z[\mathrm{M}+\mathrm{H}]^{+} 378.24$.

2-((1H-1,2,4-Triazol-3-yl)thio)-N-(6-(hexyloxy)benzo[d]thiazol-2-yl)acetamide (5e). White solid in 51.6\%. mp: $118-119{ }^{\circ} \mathrm{C} .{ }^{1} \mathrm{H}-\mathrm{NMR}(300 \mathrm{MHz}, \mathrm{DMSO}) \delta 14.10(\mathrm{~s}, 1 \mathrm{H}$, triazole $-\mathrm{NH}-), 12.45(\mathrm{~s}, 1 \mathrm{H},-\mathrm{CO}-\mathrm{NH}-)$, $8.47(\mathrm{~s}, 1 \mathrm{H}$, triazole $=\mathrm{CH}-), 7.63(\mathrm{~d}, J=8.8 \mathrm{~Hz}, 1 \mathrm{H}, \mathrm{Ar}-\mathrm{H}), 7.55(\mathrm{~d}, J=2.4 \mathrm{~Hz}, 1 \mathrm{H}, \mathrm{Ar}-\mathrm{H}), 7.01(\mathrm{dd}$, $J=8.8,2.5 \mathrm{~Hz}, 1 \mathrm{H}, \mathrm{Ar}-\mathrm{H}), 4.18\left(\mathrm{~s}, 2 \mathrm{H},-\mathrm{S}-\mathrm{CH}_{2}-\right), 3.99\left(\mathrm{t}, J=6.5 \mathrm{~Hz}, 2 \mathrm{H},-\mathrm{OCH}_{2}-\right), 1.84-1.62(\mathrm{~m}, 2 \mathrm{H}$, - $\left.\mathrm{CH}_{2}-\right), 1.51-1.11\left(\mathrm{~m}, 6 \mathrm{H},-\mathrm{CH}_{2}-\right), 0.88\left(\mathrm{t}, J=6.9 \mathrm{~Hz}, 3 \mathrm{H},-\mathrm{CH}_{3}\right) .{ }^{13} \mathrm{C}-\mathrm{NMR}(75 \mathrm{MHz}, \mathrm{DMSO}) \delta 167.41$, $155.73,155.60,145.38,145.16,142.55,132.78,121.19,115.33,105.58,68.92,35.09,30.50,28.85,28.65,22.11$, 13.66. IR $(\mathrm{KBr}) \mathrm{cm}^{-1}: 1606.09,1552.62(\mathrm{C}=\mathrm{N})$. Tof-MS: $m / z[\mathrm{M}+\mathrm{H}]^{+} 391.99$.

2-((1H-1,2,4-Triazol-3-yl)thio)-N-(6-(heptyloxy)benzo[d]thiazol-2-yl)acetamide (5f). White solid in $67.7 \%$. mp: $147-148{ }^{\circ} \mathrm{C} .{ }^{1} \mathrm{H}-\mathrm{NMR}(300 \mathrm{MHz}, \mathrm{DMSO}) \delta 14.11$ (s, $1 \mathrm{H}$, triazole -NH-), 12.45 (s, 1H, -CO-NH-), $8.57(\mathrm{~s}, 1 \mathrm{H}$, triazole $=\mathrm{CH}-), 7.63(\mathrm{~d}, J=9.1 \mathrm{~Hz}, 1 \mathrm{H}, \mathrm{Ar}-\mathrm{H}), 7.55(\mathrm{~s}, 1 \mathrm{H}, \mathrm{Ar}-\mathrm{H}), 7.02(\mathrm{dd}, J=8.8,2.5 \mathrm{~Hz}$, $1 \mathrm{H}, \mathrm{Ar}-\mathrm{H}), 4.18\left(\mathrm{~s}, 2 \mathrm{H},-\mathrm{S}-\mathrm{CH}_{2}-\right), 3.99\left(\mathrm{t}, J=6.5 \mathrm{~Hz}, 2 \mathrm{H},-\mathrm{OCH}_{2}-\right), 1.83-1.65\left(\mathrm{~m}, 2 \mathrm{H},-\mathrm{CH}_{2}-\right), 1.49-1.19$ $\left(\mathrm{m}, 8 \mathrm{H},-\mathrm{CH}_{2}-\right), 0.88\left(\mathrm{t}, J=7.0 \mathrm{~Hz}, 3 \mathrm{H},-\mathrm{CH}_{3}\right) .{ }^{13} \mathrm{C}-\mathrm{NMR}(75 \mathrm{MHz}, \mathrm{DMSO}) \delta 167.40,155.74,155.65$, $145.08,145.00,142.58,132.83,121.18,115.35,105.51,68.16,35.14,31.25,28.76,28.45,25.52,22.05,13.91$. IR $(\mathrm{KBr}) \mathrm{cm}^{-1}: 1606.15,1550.45(\mathrm{C}=\mathrm{N})$. Tof-MS: $m / z$ [M + H] $]^{+} 406.09$.

2-((1H-1,2,4-Triazol-3-yl)thio)-N-(6-(benzyloxy)benzo[d]thiazol-2-yl)acetamide (5g). White solid in 49.9\%. mp: 181-182 ${ }^{\circ} \mathrm{C} .{ }^{1} \mathrm{H}-\mathrm{NMR}(300 \mathrm{MHz}, \mathrm{DMSO}) \delta 14.12(\mathrm{~s}, 1 \mathrm{H}$, triazole $-\mathrm{NH}-), 12.49(\mathrm{~s}, 1 \mathrm{H},-\mathrm{CO}-\mathrm{NH}-)$, $8.58(\mathrm{~s}, 1 \mathrm{H}$, triazole $=\mathrm{CH}-), 7.75-7.64(\mathrm{~m}, 2 \mathrm{H}, \mathrm{Ar}-\mathrm{H}), 7.48(\mathrm{~d}, J=6.8 \mathrm{~Hz}, 2 \mathrm{H}, \mathrm{Ar}-\mathrm{H}), 7.41(\mathrm{dd}, J=11.3$, $4.4 \mathrm{~Hz}, 2 \mathrm{H}, \mathrm{Ar}-\mathrm{H}), 7.34(\mathrm{dd}, J=8.3,5.7 \mathrm{~Hz}, 1 \mathrm{H}, \mathrm{Ar}-\mathrm{H}), 7.11(\mathrm{dd}, J=8.8,2.6 \mathrm{~Hz}, 1 \mathrm{H}, \mathrm{Ar}-\mathrm{H}), 5.15(\mathrm{~s}, 2 \mathrm{H}$, $\left.-\mathrm{OCH}_{2}-\right)$, 4.19 (s, 2H, $\left.-\mathrm{S}-\mathrm{CH}_{2}-\right) .{ }^{13} \mathrm{C}-\mathrm{NMR}(75 \mathrm{MHz}, \mathrm{DMSO}) \delta 167.96,156.34,155.65,146.36,145.36$, 143.23, 140.45, 137.42, 133.15, 128.91, 128.31, 121.72, 116.01, 106.41, 70.24, 35.40. IR (KBr) cm ${ }^{-1}: 1605.62$, 1551.67 (C=N). Tof-MS: $m / z[\mathrm{M}+\mathrm{H}]^{+} 398.26$. 
2-((1H-1,2,4-Triazol-3-yl)thio)-N-(6-((2-fluorobenzyl)oxy)benzo[d]thiazol-2-yl)acetamide (5h). White solid in 58.3\%. mp: $164-165^{\circ} \mathrm{C} .{ }^{1} \mathrm{H}-\mathrm{NMR}(300 \mathrm{MHz}, \mathrm{DMSO}) \delta 14.10(\mathrm{~s}, 1 \mathrm{H}$, triazole $-\mathrm{NH}-), 12.51(\mathrm{~s}, 1 \mathrm{H}$, $-\mathrm{CO}-\mathrm{NH}-), 8.56(\mathrm{~s}, 1 \mathrm{H}$, triazole $=\mathrm{CH}-), 7.76-7.70(\mathrm{~m}, 1 \mathrm{H}), 7.69-7.64(\mathrm{~m}, 1 \mathrm{H}), 7.59(\mathrm{t}, J=7.1 \mathrm{~Hz}, 1 \mathrm{H}$, Ar-H), 7.49-7.38 (m, 1H, Ar-H), 7.32-7.21 (m, 2H, Ar-H), 7.12 (dd, J = 8.8, 2.5 Hz, 1H, Ar-H), 5.18 $\left(\mathrm{s}, 2 \mathrm{H},-\mathrm{OCH}_{2}-\right), 4.20\left(\mathrm{~s}, 2 \mathrm{H},-\mathrm{S}-\mathrm{CH}_{2}-\right) .{ }^{13} \mathrm{C}-\mathrm{NMR}(75 \mathrm{MHz}, \mathrm{DMSO}) \delta 167.96,160.90(244.5 \mathrm{~Hz}), 156.48$, $155.49,146.96,145.36,143.41,133.18,131.29,131.24,127.62,125.02,121.74,115.98$ (5.3 Hz) 115.73, 106.45, 64.64, 35.47. IR (KBr) cm ${ }^{-1}:$ 1606.04, 1552.16 (C=N). Tof-MS: $m / z[\mathrm{M}+\mathrm{H}]^{+} 415.52$.

2-((1H-1,2,4-Triazol-3-yl)thio)-N-(6-((3-fluorobenzyl)oxy)benzo[d]thiazol-2-yl)acetamide (5i). White solid in $41.6 \%$. mp: $171-172{ }^{\circ} \mathrm{C} .{ }^{1} \mathrm{H}-\mathrm{NMR}(300 \mathrm{MHz}, \mathrm{DMSO}) \delta 14.11(\mathrm{~s}, 1 \mathrm{H}$, triazole $-\mathrm{NH}-), 12.39(\mathrm{~s}, 1 \mathrm{H}$, $-\mathrm{CO}-\mathrm{NH}-), 8.52(\mathrm{~s}, 1 \mathrm{H}$, triazole $=\mathrm{CH}-), 7.75-7.60(\mathrm{~m}, 2 \mathrm{H}, \mathrm{Ar}-\mathrm{H}), 7.52-7.39(\mathrm{~m}, 1 \mathrm{H}, \mathrm{Ar}-\mathrm{H}), 7.36-7.24$ $(\mathrm{m}, 2 \mathrm{H}, \mathrm{Ar}-\mathrm{H}), 7.22-7.06(\mathrm{~m}, 2 \mathrm{H}, \mathrm{Ar}-\mathrm{H}), 5.18\left(\mathrm{~s}, 2 \mathrm{H},-\mathrm{OCH}_{2}-\right), 4.19$ (s, 2H, $\left.-\mathrm{S}-\mathrm{CH}_{2}-\right) .{ }^{13} \mathrm{C}-\mathrm{NMR}$ (75 MHz, DMSO) \& 167.82, 161.53 (243.75Hz), 156.39, 155.58, 146.32, 145.89, 143.62, 140.92, 133.28, 131.70, 123.25, 121.69, 115.82, $115.42(21.5 \mathrm{~Hz}), 115.24(6.8 \mathrm{~Hz}), 106.61,66.87,35.52 . \mathrm{IR}(\mathrm{KBr}) \mathrm{cm}^{-1}$ : 1605.21, $1553.98(\mathrm{C}=\mathrm{N})$. Tof-MS: $m / z[\mathrm{M}+\mathrm{H}]^{+} 415.68$.

2-((1H-1,2,4-Triazol-3-yl)thio)-N-(6-((4-fluorobenzyl)oxy)benzo[d]thiazol-2-yl)acetamide (5j). White solid in 43.2\%. mp: 206-207 ${ }^{\circ} \mathrm{C} .{ }^{1} \mathrm{H}-\mathrm{NMR}(300 \mathrm{MHz}, \mathrm{DMSO}) \delta 14.09$ (s, $1 \mathrm{H}$, triazole $\left.-\mathrm{NH}-\right), 12.43(\mathrm{~s}, 1 \mathrm{H}$, $-\mathrm{CO}-\mathrm{NH}-), 8.46(\mathrm{~s}, 1 \mathrm{H}$, triazole $=\mathrm{CH}-), 7.77-7.61(\mathrm{~m}, 2 \mathrm{H}, \mathrm{Ar}-\mathrm{H}), 7.58-7.42(\mathrm{~m}, 2 \mathrm{H}, \mathrm{Ar}-\mathrm{H}), 7.33-7.17$ (m, 2H, Ar-H), 7.15-7.04 (m, 1H), $5.13\left(\mathrm{~s}, 2 \mathrm{H},-\mathrm{OCH}_{2}-\right), 4.20\left(\mathrm{~s}, 2 \mathrm{H},-\mathrm{S}-\mathrm{CH}_{2}-\right) .{ }^{13} \mathrm{C}-\mathrm{NMR}(75 \mathrm{MHz}$, DMSO) $\delta 167.62,161.80(242.3 \mathrm{~Hz}) 156.72,156.15,155.08,146.10,142.88,133.19,132.76,130.08(8.3 \mathrm{~Hz})$, $121.24,115.55,115.26(\mathrm{~d}, J=21.4 \mathrm{~Hz}), 106.02,69.13,35.16 . \mathrm{IR}(\mathrm{KBr}) \mathrm{cm}^{-1}: 1604.63,1554.77(\mathrm{C}=\mathrm{N})$. Tof-MS: $m / z[\mathrm{M}+\mathrm{H}]^{+} 415.57$.

2-((1H-1,2,4-Triazol-3-yl)thio)-N-(6-((2-chlorobenzyl)oxy)benzo[d]thiazol-2-yl)acetamide (5k). White solid in 33.5\%. mp: $198-199{ }^{\circ} \mathrm{C} .{ }^{1} \mathrm{H}-\mathrm{NMR}(300 \mathrm{MHz}, \mathrm{DMSO}) \delta 14.11$ (s, $1 \mathrm{H}$, triazole $\left.-\mathrm{NH}-\right), 12.51(\mathrm{~s}, 1 \mathrm{H}$, -CO-NH-), $8.56(\mathrm{~s}, 1 \mathrm{H}$, triazole $=\mathrm{CH}-), 7.75-7.61(\mathrm{~m}, 3 \mathrm{H}, \mathrm{Ar}-\mathrm{H}), 7.58-7.50(\mathrm{~m}, 1 \mathrm{H}, \mathrm{Ar}-\mathrm{H}), 7.44-7.35$ $(\mathrm{m}, 2 \mathrm{H}, \mathrm{Ar}-\mathrm{H}), 7.13(\mathrm{dd}, J=8.8,2.4 \mathrm{~Hz}, 1 \mathrm{H}, \mathrm{Ar}-\mathrm{H}), 5.20\left(\mathrm{~s}, 2 \mathrm{H},-\mathrm{OCH}_{2}-\right), 4.20\left(\mathrm{~s}, 2 \mathrm{H},-\mathrm{S}-\mathrm{CH}_{2}-\right)$. ${ }^{13} \mathrm{C}-\mathrm{NMR}$ (75 MHz, DMSO) $\delta 167.52,156.13,155.06,146.21,144.96,143.05,134.27,132.82,132.72,130.18$, 129.87, 129.39, 127.34, 121.32, 115.48, 106.10, 67.55, 35.10. IR (KBr) cm ${ }^{-1}: 1604.77,1558.48(\mathrm{C}=\mathrm{N})$. Tof-MS: $m / z[\mathrm{M}+\mathrm{H}]^{+} 431.54$.

2-((1H-1,2,4-Triazol-3-yl)thio)-N-(6-((3-chlorobenzyl)oxy)benzo[d]thiazol-2-yl)acetamide (51). White solid in 54.3\%. mp: $172-173{ }^{\circ} \mathrm{C} .{ }^{1} \mathrm{H}-\mathrm{NMR}(300 \mathrm{MHz}, \mathrm{DMSO}) \delta 14.10(\mathrm{~s}, 1 \mathrm{H}$, triazole $-\mathrm{NH}-), 12.52(\mathrm{~m}, 1 \mathrm{H}$, $-\mathrm{CO}-\mathrm{NH}-), 8.55(\mathrm{~s}, 1 \mathrm{H}$, triazole $=\mathrm{CH}-), 7.74-7.62(\mathrm{~m}, 2 \mathrm{H}, \mathrm{Ar}-\mathrm{H}), 7.55(\mathrm{~s}, 1 \mathrm{H}, \mathrm{Ar}-\mathrm{H}), 7.50-7.34(\mathrm{~m}, 3 \mathrm{H}$, Ar-H), 7.17-7.07 (m, 1H, Ar-H), 5.17 (s, 2H, $\left.-\mathrm{OCH}_{2}-\right), 4.20$ (s, 2H, $\left.-\mathrm{S}-\mathrm{CH}_{2}-\right) .{ }^{13} \mathrm{C}-\mathrm{NMR}(75 \mathrm{MHz}$, DMSO) $\delta 167.52,156.04,154.96,145.53,145.06,142.96,139.56,133.17,132.77,130.33,127.79,127.40$, 126.24, 121.30, 115.53, 106.09, 68.91, 35.10. IR (KBr) cm ${ }^{-1}: 1605.28,1557.79(\mathrm{C}=\mathrm{N})$. Tof-MS: $\mathrm{m} / \mathrm{z}$ $[\mathrm{M}+\mathrm{H}]^{+} 431.49$.

2-((1H-1,2,4-Triazol-3-yl)thio)-N-(6-((3-(trifluoromethyl)benzyl)oxy)benzo[d]thiazol-2-yl)acetamide (5m). White solid in 46.8\%. mp: $182-183{ }^{\circ} \mathrm{C} .{ }^{1} \mathrm{H}-\mathrm{NMR}$ (300 MHz, DMSO) $\delta 14.15$ (s, $1 \mathrm{H}$, triazole $-\mathrm{NH}-$ ), $12.54(\mathrm{~s}, 1 \mathrm{H},-\mathrm{CO}-\mathrm{NH}-), 8.53(\mathrm{~s}, 1 \mathrm{H}$, triazole $=\mathrm{CH}-), 7.90-7.77(\mathrm{~m}, 2 \mathrm{H}, \mathrm{Ar}-\mathrm{H}), 7.75-7.57(\mathrm{~m}, 4 \mathrm{H}, \mathrm{Ar}-\mathrm{H})$, 7.21-7.09 (m, 1H, Ar-H), 5.26 (s, 2H, $\left.-\mathrm{OCH}_{2}-\right), 4.20$ (s, 2H, $\left.-\mathrm{S}-\mathrm{CH}_{2}-\right) .{ }^{13} \mathrm{C}-\mathrm{NMR}$ (75 MHz, DMSO) ס 167.51, 156.09, 154.99, 146.22, 146.20, 145.86, 144.67, 143.05, 138.58, 132.80, 131.71, 129.56, 129.06, $124.32(\mathrm{dd}, J=36.0,3.3 \mathrm{~Hz}) .121 .31,115.55,106.26,69.08,35.14 . \mathrm{IR}(\mathrm{KBr}) \mathrm{cm}^{-1}: 1606.27,1556.18(\mathrm{C}=\mathrm{N})$. Tof-MS: $m / z[\mathrm{M}+\mathrm{H}]^{+} 465.42$. 
3.2.5. General Procedure for the Synthesis of N-(6-Alkoxybenzo[d]thiazol-2-yl)-2-(1H-imidazol-1-yl) acetamide $(\mathbf{6 a}-\mathbf{b})$

A solution of 2-chloro- $N$-(6-alkoxybenzo[d]thiazol-2-yl)acetamide ( $5 \mathrm{mmol})$, imidazole (6 mmol), and sodium hydroxide $(6 \mathrm{mmol})$ in $N, N$-dimethylformamide $(10 \mathrm{~mL})$ was stirred at room temperature for $12 \mathrm{~h}$. Half of the solvent was then evaporated and the solution was poured in $50 \mathrm{~mL}$ water and then extracted with ethyl acetate $(30 \mathrm{~mL} \times 3)$. The ethyl acetate layer was dried over anhydrous $\mathrm{MgSO}_{4}$. Evaporation of the solvent yielded a crude product, which was recrystallized from dichloromethane to yield a white solid.

N-(6-((3-Fluorobenzyl)oxy)benzo[d]thiazol-2-yl)-2-(1H-imidazol-1-yl)acetamide (6a). White solid in 47.1\%. mp: $224-225^{\circ} \mathrm{C} .{ }^{1} \mathrm{H}-\mathrm{NMR}(300 \mathrm{MHz}, \mathrm{DMSO}) \delta 12.55$ (s, 1H, -CO-NH-), 7.72-7.62 (m, 3H, imidazole-H and $\mathrm{Ar}-\mathrm{H}), 7.45(\mathrm{dd}, J=14.3,7.4 \mathrm{~Hz}, 1 \mathrm{H}, \mathrm{Ar}-\mathrm{H}), 7.35-7.26(\mathrm{~m}, 2 \mathrm{H}, \mathrm{Ar}-\mathrm{H}), 7.24-7.08(\mathrm{~m}, 3 \mathrm{H}$, imidazole-H and $\mathrm{Ar}-\mathrm{H}), 6.91\left(\mathrm{~s}, 1 \mathrm{H}\right.$, imidazole-H), $5.17\left(\mathrm{~s}, 2 \mathrm{H},-\mathrm{OCH}_{2}-\right), 5.08\left(\mathrm{~s}, 2 \mathrm{H}\right.$, imidazole $\left.-\mathrm{CH}_{2}-\right) .{ }^{13} \mathrm{C}-\mathrm{NMR}$ (75 MHz, DMSO) $\delta 166.89,162.56(\mathrm{~d}, J=243.5 \mathrm{~Hz}), 156.02,155.63,143.32,140.38(\mathrm{~d}, J=7.5 \mathrm{~Hz})$, $138.96,133.19,130.89(\mathrm{~d}, J=8.3 \mathrm{~Hz}), 128.58,124.56,121.87,121.35,116.08,115.12(\mathrm{~d}, J=21.2 \mathrm{~Hz})$, $114.95(\mathrm{~d}, J=22.5 \mathrm{~Hz}), 106.61,69.46,49.89$. IR $(\mathrm{KBr}) \mathrm{cm}^{-1}: 1604.77,1546.91(\mathrm{C}=\mathrm{N})$. Tof-MS: $\mathrm{m} / \mathrm{z}$ $[\mathrm{M}+\mathrm{H}]^{+} 383.05$.

N-(6-((4-Fluorobenzyl)oxy)benzo[d]thiazol-2-yl)-2-(1H-imidazol-1-yl)acetamide (6b). White solid in 56.0\%. mp: $246-247{ }^{\circ} \mathrm{C} .{ }^{1} \mathrm{H}-\mathrm{NMR}(300 \mathrm{MHz}, \mathrm{DMSO}) \delta 12.52$ (s, 1H,-CO-NH-), 7.73-7.63 (m, 3H, imidazole-H and Ar-H), 7.60-7.46 (m, 2H, Ar-H), 7.27-7.19 (m, 2H, Ar-H), 7.14-7.08 (m, 1H, Ar-H), 7.04 (s, 1H, imidazole-H), $6.92\left(\mathrm{~s}, 1 \mathrm{H}\right.$, imidazole-H), $5.12\left(\mathrm{~s}, 2 \mathrm{H},-\mathrm{OCH}_{2}-\right), 5.09\left(\mathrm{~s}, 2 \mathrm{H}\right.$, imidazole- $\left.\mathrm{CH}_{2}-\right) .{ }^{13} \mathrm{C}-\mathrm{NMR}$ (75 MHz, DMSO) $\delta 167.49,162.26(\mathrm{~d}, J=243.7 \mathrm{~Hz}), 156.18,155.61,143.23,138.88,133.63,133.18,130.53$ $(\mathrm{d}, J=8.3 \mathrm{~Hz}), 128.46,121.77,121.37,116.09,115.72(\mathrm{~d}, J=21.4 \mathrm{~Hz}), 106.51,69.56,48.99 . \mathrm{IR}(\mathrm{KBr}) \mathrm{cm}^{-1}$ : 1604.95, 1557.26 (C=N). Tof-MS: $m / z[\mathrm{M}+\mathrm{H}]^{+} 383.00$.

3.2.6. General Procedure for the Synthesis of N-(6-Alkoxybenzo[d]thiazol-2-yl)-2-(1H-1,2,4-triazol-1-yl) acetamide $(7 \mathbf{a}-\mathbf{b})$

2-Chloro- $\mathrm{N}$-(6-alkoxybenzo[d] thiazol-2-yl)acetamide (5 mmol) and sodium 1,2,4-triazole (6 mmol) were dissolved in $\mathrm{N}, \mathrm{N}$-dimethylformamide $(10 \mathrm{~mL})$ and stirred for $10 \mathrm{~h}$ at room temperature. Half of the solvent was then evaporated, the solution was poured in $50 \mathrm{~mL}$ water, and extracted with ethyl acetate $(30 \mathrm{~mL} \times 3)$. The ethyl acetate layer was dried over anhydrous $\mathrm{MgSO}_{4}$. After removing the solvent under reduced pressure, the crude product was obtained and recrystallized from dichloromethane to yield a white solid.

N-(6-((3-Fluorobenzyl)oxy)benzo[d]thiazol-2-yl)-2-(1H-1,2,4-triazol-1-yl)acetamide (7a). White solid in 55.1\%. mp: $212-213{ }^{\circ} \mathrm{C} .{ }^{1} \mathrm{H}-\mathrm{NMR}(300 \mathrm{MHz}, \mathrm{DMSO}) \delta 12.73$ (s, $\left.1 \mathrm{H},-\mathrm{CO}-\mathrm{NH}-\right), 8.59$ (s, $1 \mathrm{H}$, triazole-H), $8.03(\mathrm{~s}, 1 \mathrm{H}$, triazole-H), 7.77-7.64 (m, 2H, Ar-H), 7.52-7.40 (m, 1H, Ar-H), 7.37-7.27 (m, 2H, Ar-H), 7.23-7.10 (m, 2H, Ar-H), $5.33\left(\mathrm{~s}, 2 \mathrm{H}\right.$, triazole $\left.-\mathrm{CH}_{2}-\right), 5.18\left(\mathrm{~s}, 2 \mathrm{H},-\mathrm{OCH}_{2}-\right) .{ }^{13} \mathrm{C}-\mathrm{NMR}(75 \mathrm{MHz}, \mathrm{DMSO})$ $\delta 166.31,162.67(\mathrm{~d}, J=243.8 \mathrm{~Hz}), 156.09,155.57,152.04,146.23,142.99,140.38(\mathrm{~d}, J=7.5 \mathrm{~Hz}), 133.21$, $130.94(\mathrm{~d}, J=8.4 \mathrm{~Hz}), 124.07(\mathrm{~d}, J=2.6 \mathrm{~Hz}), 121.88,116.15,115.08(\mathrm{~d}, J=21.0 \mathrm{~Hz}), 114.77(\mathrm{~d}, J=21.8 \mathrm{~Hz})$, 106.59, 69.42, 51.57. IR (KBr) cm ${ }^{-1}: 1605.72,1556.78(\mathrm{C}=\mathrm{N})$. Tof-MS: $m / z[\mathrm{M}+\mathrm{H}]^{+} 383.65$.

N-(6-((4-Fluorobenzyl)oxy)benzo[d]thiazol-2-yl)-2-(1H-1,2,4-triazol-1-yl)acetamide (7b). White solid in 39.5\%. mp: $227-228{ }^{\circ} \mathrm{C} .{ }^{1} \mathrm{H}-\mathrm{NMR}(300 \mathrm{MHz}, \mathrm{DMSO}) \delta 12.72(\mathrm{~s}, 1 \mathrm{H},-\mathrm{CO}-\mathrm{NH}-), 8.59$ (s, $1 \mathrm{H}$, triazole-H), $8.04(\mathrm{~s}, 1 \mathrm{H}$, triazole-H), $7.69(\mathrm{dd}, J=5.6,3.0 \mathrm{~Hz}, 2 \mathrm{H}, \mathrm{Ar}-\mathrm{H}), 7.53(\mathrm{dd}, J=8.5,5.7 \mathrm{~Hz}, 2 \mathrm{H}, \mathrm{Ar}-\mathrm{H}), 7.23$ $(\mathrm{t}, J=8.9 \mathrm{~Hz}, 2 \mathrm{H}, \mathrm{Ar}-\mathrm{H}), 7.12(\mathrm{dd}, J=8.9,2.5 \mathrm{~Hz}, 1 \mathrm{H}, \mathrm{Ar}-\mathrm{H}), 5.33\left(\mathrm{~s}, 2 \mathrm{H}\right.$, triazole $\left.-\mathrm{CH}_{2}-\right), 5.13(\mathrm{~s}, 2 \mathrm{H}$, $\left.-\mathrm{OCH}_{2}-\right) .{ }^{13} \mathrm{C}-\mathrm{NMR}(75 \mathrm{MHz}, \mathrm{DMSO}) \delta 166.26,162.27(\mathrm{~d}, J=242.25 \mathrm{~Hz}), 155.92,155.70,152.04,146.22$, $143.31,133.65,133.23,130.54(\mathrm{~d}, J=8.3 \mathrm{~Hz}), 121.89,115.72(\mathrm{~d}, J=21.4 \mathrm{~Hz}), 115.58,106.52,69.56,51.56$. IR (KBr) cm ${ }^{-1}: 1605.71,1556.18(\mathrm{C}=\mathrm{N})$. Tof-MS: $m / z[\mathrm{M}+\mathrm{H}]^{+} 383.57$. 
3.2.7. General Procedure for the Synthesis of N-(6-Alkoxybenzo[d]thiazol-2-yl)-2-(1H-tetrazol-1-yl) acetamide $(\mathbf{8} \mathbf{a}-\mathbf{b})$

A mixture of 2-chloro- $N$-(6-alkoxybenzo[d]thiazol-2-yl)acetamide ( $5 \mathrm{mmol}), 1 \mathrm{H}$-tetrazole $(6 \mathrm{mmol})$, and sodium hydroxide $(6 \mathrm{mmol})$ in $N, N$-dimethylformamide $(10 \mathrm{~mL})$ was stirred at room temperature for $10 \mathrm{~h}$. The solution was then poured in $50 \mathrm{~mL}$ of water and extracted with ethyl acetate $(30 \mathrm{~mL} \times 3)$. The ethyl acetate layer was dried over anhydrous $\mathrm{MgSO}_{4}$. Evaporation of the solvent provided a crude product, which was recrystallized from dichloromethane to obtain a white solid.

$N-(6-((3-F l u o r o b e n z y l) o x y)$ benzo[d]thiazol-2-yl)-2-(1H-tetrazol-1-yl)acetamide (8a). White solid in 48.3\%. mp: $205-206{ }^{\circ} \mathrm{C} .{ }^{1} \mathrm{H}-\mathrm{NMR}(300 \mathrm{MHz}, \mathrm{DMSO}) \delta 12.86$ (s, 1H, -CO-NH-), 9.45 (s, 1H, tetrazole-H), 7.76-7.65 (m, 2H, Ar-H), 7.49-7.40 (m, 1H, Ar-H), 7.32 (s, 1H, Ar-H), 7.30 (s, 1H, Ar-H), 7.21-7.11 $(\mathrm{m}, 2 \mathrm{H}, \mathrm{Ar}-\mathrm{H}), 5.67\left(\mathrm{~s}, 2 \mathrm{H}\right.$, tetrazole- $\left.\mathrm{CH}_{2}-\right), 5.18\left(\mathrm{~s}, 2 \mathrm{H},-\mathrm{OCH}_{2}-\right) .{ }^{13} \mathrm{C}-\mathrm{NMR}(75 \mathrm{MHz}, \mathrm{DMSO}) \delta 165.43$, $162.66(\mathrm{~d}, J=243.6 \mathrm{~Hz}), 156.18,155.60,145.76,143.13,140.36(\mathrm{~d}, J=7.5 \mathrm{~Hz}), 133.18,130.95(\mathrm{~d}, J=8.3 \mathrm{~Hz})$, $124.10,121.88,116.19,115.10(\mathrm{~d}, J=21.2 \mathrm{~Hz}), 114.78(\mathrm{~d}, J=22.1 \mathrm{~Hz}), 106.62,69.43,50.11 . \mathrm{IR}(\mathrm{KBr}) \mathrm{cm}^{-1}$ : 1606.36, $1556.98(\mathrm{C}=\mathrm{N})$. Tof-MS: $m / z[\mathrm{M}+\mathrm{H}]^{+} 382.87$.

N-(6-((4-Fluorobenzyl)oxy)benzo[d]thiazol-2-yl)-2-(1H-tetrazol-1-yl)acetamide (8b). White solid in 68.4\%. mp: $224-225^{\circ} \mathrm{C} .{ }^{1} \mathrm{H}-\mathrm{NMR}(300 \mathrm{MHz}, \mathrm{DMSO}) \delta 12.86(\mathrm{~s}, 1 \mathrm{H},-\mathrm{CO}-\mathrm{NH}-), 9.46(\mathrm{~s}, 1 \mathrm{H}$, tetrazole-H), $7.69(\mathrm{~s}, 2 \mathrm{H}, \mathrm{Ar}-\mathrm{H}), 7.59-7.47(\mathrm{~m}, 2 \mathrm{H}, \mathrm{Ar}-\mathrm{H}), 7.23(\mathrm{t}, J=8.6 \mathrm{~Hz}, 2 \mathrm{H}, \mathrm{Ar}-\mathrm{H}), 7.13(\mathrm{dd}, J=9.0,1.6 \mathrm{~Hz}$, $1 \mathrm{H}, \mathrm{Ar}-\mathrm{H}), 5.68\left(\mathrm{~s}, 2 \mathrm{H}\right.$, tetrazole- $\left.\mathrm{CH}_{2}-\right), 5.13\left(\mathrm{~s}, 2 \mathrm{H},-\mathrm{OCH}_{2}-\right) .{ }^{13} \mathrm{C}-\mathrm{NMR}(75 \mathrm{MHz}, \mathrm{DMSO}) \delta 166.30$, $162.26(\mathrm{~d}, J=243.5 \mathrm{~Hz}), 156.09,155.58,145.76,143.13,133.66,133.23,130.60(\mathrm{~d}, J=8.3 \mathrm{~Hz}), 121.90$, $116.09,115.69(\mathrm{~d}, J=21.2 \mathrm{~Hz}), 106.60,69.56,50.95$. IR (KBr) cm ${ }^{-1}: 1606.01,1555.99$ (C=N). Tof-MS: $\mathrm{m} / \mathrm{z}$ $[\mathrm{M}+\mathrm{H}]^{+} 382.99$.

3.2.8. General Procedure for the Synthesis of 2-(3-Amino-1H-1,2,4-triazol-1-yl)-N-(6-alkoxybenzo[d] thiazol-2-yl)acetamide $(\mathbf{9 a}-\mathbf{b})$

$1 H$-1,2,4-triazol-3-amine (6 mmol), 2-chloro- $\mathrm{N}$-(6-alkoxybenzo[d]thiazol-2-yl) acetamide (5 mmol), and sodium hydroxide $(6 \mathrm{mmol})$ in $N, N$-dimethylformamide $(10 \mathrm{~mL})$ were dissolved and stirred at room temperature for $8 \mathrm{~h}$. $50 \mathrm{~mL}$ of water was added to the reaction mixture, which was then extracted with ethyl acetate $(30 \mathrm{~mL} \times 3)$. The combined layer of ethyl acetate was dried over anhydrous $\mathrm{MgSO}_{4}$. After removing the solvent under reduced pressure, the crude product was obtained and then recrystallized from dichloromethane to yield a white solid.

2-(3-Amino-1H-1,2,4-triazol-1-yl)-N-(6-((3-fluorobenzyl)oxy)benzo[d]thiazol-2-yl)acetamide (9a). White solid in 66.1\%. mp: $235-236{ }^{\circ} \mathrm{C} .{ }^{1} \mathrm{H}-\mathrm{NMR}(300 \mathrm{MHz}, \mathrm{DMSO}) \delta 12.58(\mathrm{~s}, 1 \mathrm{H},-\mathrm{CO}-\mathrm{NH}-), 7.70(\mathrm{~s}, 1 \mathrm{H}$, triazole-H), 7.69-7.65 (m, 1H, Ar-H), 7.50-7.41 (m, 1H, Ar-H), $7.37(\mathrm{~s}, 1 \mathrm{H}, \mathrm{Ar}-\mathrm{H}), 7.34-7.26(\mathrm{~m}, 2 \mathrm{H}$, $\mathrm{Ar}-\mathrm{H}), 7.21-7.09(\mathrm{~m}, 2 \mathrm{H}, \mathrm{Ar}-\mathrm{H}), 6.33\left(\mathrm{~s}, 2 \mathrm{H}\right.$, triazole- $\left.\mathrm{NH}_{2}\right), 5.18\left(\mathrm{~s}, 2 \mathrm{H},-\mathrm{OCH}_{2}-\right), 4.93(\mathrm{~s}, 2 \mathrm{H}$, triazole - $\left.\mathrm{CH}_{2}-\right) .{ }^{13} \mathrm{C}-\mathrm{NMR}(75 \mathrm{MHz}, \mathrm{DMSO}) \delta 166.72,162.67(\mathrm{~d}, J=243.7 \mathrm{~Hz}), 156.25,155.49,146.61,145.55$, $143.32,140.39(\mathrm{~d}, J=7.4 \mathrm{~Hz}), 133.19,130.93(\mathrm{~d}, J=8.3 \mathrm{~Hz}), 124.06(\mathrm{~d}, J=2.6 \mathrm{~Hz}), 121.80,116.07,115.07$ $(\mathrm{d}, J=21.3 \mathrm{~Hz}), 114.76(\mathrm{~d}, J=21.9 \mathrm{~Hz}), 106.57,69.42,49.12 . \mathrm{IR}(\mathrm{KBr}) \mathrm{cm}^{-1}: 1604.63,1557.39(\mathrm{C}=\mathrm{N})$. Tof-MS: $m / z[\mathrm{M}+\mathrm{H}]^{+} 398.68$.

2-(3-Amino-1H-1,2,4-triazol-1-yl)-N-(6-((4-fluorobenzyl)oxy)benzo[d] thiazol-2-yl)acetamide (9b). White solid in 60.2\%. mp: $249-250{ }^{\circ} \mathrm{C} .{ }^{1} \mathrm{H}-\mathrm{NMR}(300 \mathrm{MHz}, \mathrm{DMSO}) \delta 12.51(\mathrm{~s}, 1 \mathrm{H},-\mathrm{CO}-\mathrm{NH}-), 7.72-7.64(\mathrm{~m}, 2 \mathrm{H}$, tetrazole-H and Ar-H), 7.57-7.48 (m, 2H, Ar-H), $7.37(\mathrm{~s}, 1 \mathrm{H}, \mathrm{Ar}-\mathrm{H}), 7.23(\mathrm{t}, J=8.7 \mathrm{~Hz}, 2 \mathrm{H}, \mathrm{Ar}-\mathrm{H})$, $7.11(\mathrm{~d}, J=9.0 \mathrm{~Hz}, 1 \mathrm{H}, \mathrm{Ar}-\mathrm{H}), 6.34\left(\mathrm{~s}, 2 \mathrm{H}\right.$, triazole- $\left.\mathrm{NH}_{2}\right), 5.12\left(\mathrm{~s}, 2 \mathrm{H},-\mathrm{OCH}_{2}-\right), 4.93(\mathrm{~s}, 2 \mathrm{H}$, triazole - $\left.\mathrm{CH}_{2}-\right) .{ }^{13} \mathrm{C}-\mathrm{NMR}(75 \mathrm{MHz}$, DMSO) $\delta 167.26,162.68(\mathrm{~d}, J=243.8 \mathrm{~Hz}), 156.25,155.58,146.81,145.69$, $143.23,133.68,133.20,130.93(\mathrm{~d}, J=8.3 \mathrm{~Hz}), 121.80,116.08,115.70(\mathrm{~d}, J=21.3 \mathrm{~Hz}), 106.60,69.55,50.18$. IR $(\mathrm{KBr}) \mathrm{cm}^{-1}: 1605.88,1556.08(\mathrm{C}=\mathrm{N})$. Tof-MS: $m / z[\mathrm{M}+\mathrm{H}]^{+} 398.85$. 


\subsection{Pharmacology}

All compounds were evaluated for anticonvulsant activities with KunMing mice in the 18-22 $g$ weight range purchased from the Laboratory of Animal Research, College of Pharmacy, Yanbian University. The animals were maintained on a $12 \mathrm{~h}$ light/dark cycle and allowed free access to food and water, except during the time they were removed from their cages for testing. The experimental substances were dissolved in dimethylsulfoxide (DMSO) and administered intraperitoneally (i.p.) in a volume of $0.1 \mathrm{~mL} / 20 \mathrm{~g}$ body weight. The test method with reference to the Antiepileptic Drug Development (ADD) program [29,30].

\subsubsection{MES Screening Test}

Seizures were elicited with a $60-\mathrm{Hz}$ alternating current of $50 \mathrm{~mA}$ intensity applied via corneal electrodes for $0.2 \mathrm{~s}$. Protection against the spread of MES-induced seizures was defined as the abolition of the hind leg, and tonic maximal extension component of the seizure. The MES test was performed at $30 \mathrm{~min}$ after compound administration.

\subsubsection{ScPTZ Seizures Screening Test}

At $0.5 \mathrm{~h}$ after the administration of the test compound, $100 \mathrm{mg} / \mathrm{kg}$ PTZ (which $100 \%$ of the animals showed clonic seizure) dissolved in saline was administered subcutaneously. The animals were placed in individual cages and observed for $0.5 \mathrm{~h}$. The median effective doses $\left(E D_{50}\right)$, which showed $50 \%$ protection, were recorded.

\subsubsection{Neurotoxicity Screening Test}

The neurotoxicity of the compounds was measured in mice using the rotarod test. Mice were tested on a knurled plastic rod (diameter $3.2 \mathrm{~cm}$ ) rotating at $6 \mathrm{rpm}$ for $1 \mathrm{~min}$, at $30 \mathrm{~min}$ after compound administration. Neurotoxicity was measured by the inability of the animal to maintain equilibrium on the rod for at least 1 minute in each of the trials.

\section{Conclusions}

In the present study, we described the synthesis and anticonvulsant activity evaluation of 2-((1H-1,2,4-triazol-3-yl)thio)- $N$-(6-alkoxybenzo[d]thiazol-2-yl) acetamide (5a-m). In addition, several series of derivatives were also synthesized and evaluated for their anticonvulsant activity for SAR studies, which included $N$-(6-alkoxybenzo[d]thiazol-2-yl)-2-(1H-imidazol-1-yl)acetamide (6a-b), $N$-(6-alkoxybenzo[d]thiazol-2-yl)-2-(1H-1,2,4-triazol-1-yl)acetamide (7a-b), $N$-(6-alkoxybenzo[d]thiazol2-yl) -2-(1H-tetrazol-1-yl)acetamide (8a-b), and 2-(3-amino-1H-1,2,4-triazol-1-yl)-N-(6-alkoxybenzo[d]thiazol -2-yl)acetamide $(\mathbf{9 a}-\mathbf{b})$. All the compounds were tested for their anticonvulsant activity and neurotoxicity using the MES and rotarod tests at the dose of $100 \mathrm{mg} / \mathrm{kg}$ after i.p. Bioevaluation demonstrated that the compounds possessing 1,2,4-triazole-3-thiol displayed the best anticonvulsant activity and favorable PI. Among them, compounds $5 \mathbf{i}$ and $5 \mathbf{j}$ were the most potent, with an $\mathrm{ED}_{50}$ value of $50.8 \mathrm{mg} / \mathrm{kg}$ and $54.8 \mathrm{mg} / \mathrm{kg}$ in the MES test and $76.0 \mathrm{mg} / \mathrm{kg}$ and $52.8 \mathrm{mg} / \mathrm{kg}$ in the scPTZ seizures test, respectively. They also presented markedly decreased neurotoxicity and, therefore, a higher protective index. Especially, compound $5 \mathbf{j}$ showed high PI values of 8.96 in the MES test and 9.30 in the scPTZ test, which were better than the standard drugs, carbamazepine and valproic acid, used as positive controls in this study.

Acknowledgments: This work was supported by the National Natural Science Foundation of China (No. 81160382 and No. 81160409) and National Science and Technology Major Project of China (No. 2012ZX09103-101-044).

Author Contributions: Zhe-Shan Quan designed the compounds, planned synthetic pathways, Da-Chuan Liu performed chemical synthesis, biological testing, analyzed the results of biological testing and wrote the manuscript. Chun-Mei Jin and Hong-Jian Zhang performed part of chemical synthesis and biological testing. All authors commented on the manuscript. 
Conflicts of Interest: The authors declare no conflict of interest.

\section{References}

1. Moshé, S.L.; Perucca, E.; Ryvlin, P.; Tomson, T. Epilepsy: New advances. Lancet 2015, 385, 884-898. [CrossRef]

2. Van Nieuwenhuyse, B.; Raedt, R.; Sprengers, M.; Dauwe, I.; Gadeyne, S.; Carrette, E.; Delbeke, J.; Wadman, W.J.; Boon, P.; Vonck, K. The systemic kainic acid rat model of temporal lobe epilepsy: Long-term EEG monitoring. Brain Res. 2015, 1627, 1-11. [CrossRef] [PubMed]

3. The Lancet. A voice for people with epilepsy. Lancet 2015, 385, 482.

4. Deng, X.Q.; Song, M.X.; Zheng, Y.; Quan, Z.S. Design, synthesis and evaluation of the antidepressant and anticonvulsant activities of triazole-containing quinolinones. Eur. J. Med. Chem. 2014, 73, 217-224. [CrossRef] [PubMed]

5. Sarigol, D.; Uzgoren-Baran, A.; Tel, B.C.; Somuncuoglu, E.I.; Kazkayasi, I.; Ozadali-Sari, K.; Unsal-Tan, O.; Okay, G.; Ertan, M.; Tozkoparan, B. Novel thiazolo[3,2-b]-1,2,4-triazoles derived from naproxen with analgesic/anti-inflammatory properties: Synthesis; biological evaluation and molecular modeling studies. Bioorg. Med. Chem. 2015, 23, 2518-2528. [CrossRef] [PubMed]

6. Pandey, R.; Ahmad, Z.; Sharma, S.; Khuller, G.K. Nano-encapsulation of azole antifungals: Potential applications to improve oral drug delivery. Int. J. Pharm. 2005, 301, 268-276. [CrossRef] [PubMed]

7. Barradas, J.S.; Errea, M.I.; D’Accorso, N.B.; Sepúlveda, C.S.; Talarico, L.B.; Damonte, E.B. Synthesis and antiviral activity of azoles obtained from carbohydrates. Carbohydr. Res. 2008, 343, 2468-2474. [CrossRef] [PubMed]

8. Tyagi, P.; Chandra, S.; Saraswat, B.S.; Yadav, D. Design, spectral characterization; thermal; DFT studies and anticancer cell line activities of $\mathrm{Co}(\mathrm{II}) ; \mathrm{Ni}(\mathrm{II})$ and $\mathrm{Cu}(\mathrm{II})$ complexes of Schiff bases derived from 4-amino-5-(pyridin-4-yl) 4H-1;2;4-triazole-3-thiol. Spectrochim. Acta A Mol. Biomol. Spectrosc. 2015, 145, 155-164. [CrossRef] [PubMed]

9. Khanmohammadi, H.; Abnosi, M.H.; Hosseinzadeh, A. Synthesis, biological and computational study of new Schiff base hydrazones bearing 3-(4-pyridine)-5-mercapto-1,2,4-triazole moiety. Spectrochim. Acta A Mol. Biomol. Spectrosc. 2008, 71, 1474-1480. [CrossRef] [PubMed]

10. Hussein, W.M.; Vella, P.; Islam, N.U.; Ollis, D.L.; Schenk, G.; McGeary, R.P. 3-Mercapto-1,2,4-triazoles and $N$-acylated thiosemicarbazides as metallo- $\beta$-lactamase inhibitors. Bioorg. Med. Chem. Lett. 2012, 22, 380-386.

11. Agarwal, S.; Agarwal, D.K.; Gautam, N.; Agarwal, K.; Gautam, D.C. Synthesis and in vitro antimicrobial evaluation of benzothiazole incorporated thiazolidin-4-ones derivatives. J. Korean Chem. Soc. 2014, 58, 33-38. [CrossRef]

12. Singh, M.K.; Tilak, R.; Nath, G.; Awasthi, S.K.; Agarwal, A. Design, synthesis and antimicrobial activity of novel benzothiazole analogs. Eur. J. Med. Chem. 2013, 63, 635-644. [CrossRef] [PubMed]

13. Stone, E.L.; Citossi, F.; Singh, R.; Kaur, B.; Gaskell, M.; Farmer, P.B.; Monks, A.; Hose, C.; Stevens, M.F.; Leong, C.O.; et al. Antitumour benzothiazoles. Part 32: DNA adducts and double strand breaks correlate with activity; synthesis of 5F203 hydrogels for local delivery. Bioorg. Med. Chem. 2015, 23, 6891-6899. [CrossRef] [PubMed]

14. Ma, J.; Bao, G.; Wang, L.; Li, W.; Xu, B.; Du, B.; Lv, J.; Zhai, X.; Gong, P. Design, synthesis, biological evaluation and preliminary mechanism study of novel benzothiazole derivatives bearing indole-based moiety as potent antitumor agents. Eur. J. Med. Chem. 2015, 96, 173-186. [CrossRef] [PubMed]

15. Ugale, V.G.; Patel, H.M.; Wadodkar, S.G.; Bari, S.B.; Shirkhedkar, A.A.; Surana, S.J. Quinazolino-benzothiazoles: Fused pharmacophores as anticonvulsant agents. Eur. J. Med. Chem. 2012, 53, 107-113. [CrossRef] [PubMed]

16. Siddiqui, N.; Rana, A.; Khan, S.A.; Bhat, M.A.; Haque, S.E. Synthesis of benzothiazole semicarbazones as novel anticonvulsants-The role of hydrophobic domain. Bioorg. Med. Chem. Lett. 2007, 17, 4178-4182. [CrossRef] [PubMed]

17. Hassan, M.Z.; Khan, S.A.; Amir, M. Design; synthesis and evaluation of N-(substituted benzothiazol-2-yl)amides as anticonvulsant and neuroprotective. Eur. J. Med. Chem. 2012, 58, 206-213. [CrossRef] [PubMed]

18. Tripathi, L.; Kumar, P.; Singh, R.; Stables, J.P. Design, synthesis and anticonvulsant evaluation of novel $N$-(4-substituted phenyl)-2-[4-(substituted) benzylidene]-hydrazinecarbothio amides. Eur. J. Med. Chem. 2012, 47, 153-166. [CrossRef] [PubMed] 
19. Liu, D.C.; Deng, X.Q.; Wang, S.B.; Quan, Z.S. Synthesis and anticonvulsant activity evaluation of 7-alkoxy[1,2,4]triazolo[3,4-b]benzothiazol-3(2H)-ones. Arch. Pharm. Chem. Life Sci. 2014, 347, 268-275. [CrossRef] [PubMed]

20. Behbehani, H.; Ibrahim, H.M. 4-Thiazolidinones in heterocyclic synthesis: Synthesis of novel enaminones, azolopyrimidines and 2-arylimino-5-arylidene-4-thiazolidinones. Molecules 2012, 17, 6362-6385. [CrossRef] [PubMed]

21. Abdelazeem, A.H.; Gouda, A.M.; Omar, H.A.; Tolba, M.F. Design; synthesis and biological evaluation of novel diphenylthiazole-based cyclooxygenase inhibitors as potential anticancer agents. Bioorg. Chem. 2014, 57, 132-141. [CrossRef] [PubMed]

22. Zhang, Y.L.; Yang, K.W.; Zhou, Y.J.; La Curan, A.E.; Oelschlaeger, P.; Crowder, M.W. Diaryl-substituted azolylthioacetamides: Inhibitor discovery of New Delhi metallo- $\beta$-lactamase-1 (NDM-1). ChemMedChem 2014, 9, 2445-2448. [CrossRef] [PubMed]

23. Yang, G.F.; Xu, L.; Lu, A.H. Synthesis and bioactivity of novel triazolo $[1,5-a]$ pyrimidine derivatives. Heteroatom Chem. 2001, 12, 491-496. [CrossRef]

24. Deb, P.K.; Kaur, R.; Chandrasekaran, B.; Bala, M.; Gill, D.; Kaki, V.R.; Akkinepalli, R.R.; Mailavaram, R. Synthesis, anti-inflammatory evaluation, and docking studies of some new thiazole derivatives. Med. Chem. Res. 2014, 23, 2780-2792. [CrossRef]

25. Papadopoulou, M.V.; Bloomer, W.D.; Rosenzweig, H.S.; Chatelain, E.; Kaiser, M.; Wilkinson, S.R.; McKenzie, C.; Ioset, J.R. Novel 3-nitro-1H-1,2,4-triazole- based amides and sulfonamides as potential antitrypanosomal agents. J. Med. Chem. 2012, 55, 5554-5565. [CrossRef] [PubMed]

26. Rogawski, M.A. Molecular targets versus models for new antiepileptic drug discovery. Epilepsy Res. 2006, 68, 22-28. [CrossRef] [PubMed]

27. Castel-Branco, M.M.; Alves, G.L.; Figueiredo, I.V.; Falcão, A.C.; Caramona, M.M. The maximal electroshock seizure (MES) model in the preclinical assessment of potential new antiepileptic drugs. Methods Find. Exp. Clin. Pharmacol. 2009, 31, 101-106. [CrossRef] [PubMed]

28. Löscher, W. Critical review of current animal models of seizures and epilepsy used in the discovery and development of new antiepileptic drugs. Seizure 2011, 20, 359-368. [CrossRef] [PubMed]

29. Krall, R.L.; Penry, J.K.; White, B.G.; Kupferberg, H.J.; Swinyard, E.A. Antiepileptic drug development: II. Anticonvulsant drug screening. Epilepsia 1978, 19, 409-428. [CrossRef] [PubMed]

30. Porter, R.J.; Cereghino, J.J.; Gladding, G.D.; Hessie, B.J.; Kupferberg, H.J.; Scoville, B.; White, B.G. Antiepileptic Drug Development Program. Cleve. Clin. Q. 1984, 51, 293-305. [CrossRef] [PubMed]

Sample Availability: Samples of all the target compounds are available from the authors.

(C) 2016 by the authors; licensee MDPI, Basel, Switzerland. This article is an open access article distributed under the terms and conditions of the Creative Commons by Attribution (CC-BY) license (http://creativecommons.org/licenses/by/4.0/). 